\title{
Improved Point Distribution Model with Curvature for Boundary Finding
}

\author{
Hae-Chul Choi ${ }^{* a}$, Seung-Dae Kim ${ }^{\mathrm{b}}$ \\ ${ }^{a}$ bept. of EE. Korea Advanced Institute of Science and Technology (KAIST), Taejon, Korea
}

\begin{abstract}
This paper proposes an algorithm for extracting the boundary of an object. In order to take full advantage of global shape, our approach uses global shape parameters derived from Point Distribution Model (PDM). Unlike PDM, the proposed method models global shape using curvature as well as edge. The objective function for applying the shape model is formulated using Bayesian rule. This method can extract a boundary of an object by evaluating the solution maximizing the objective function iteratively. Experimental results show that the proposed method requires less computational cost than the PDM and it is robust to noise, pose variation, and some occlusion.
\end{abstract}

Keywords: Point Distribution Model, curvature, boundary finding, segmentation, Infrared image, model-based segmentation

\section{INTRODUCTION}

In this paper, we try to solve the problem of extracting the boundary of an object. A shape described by a boundary is a powerful feature and the boundary extraction is one of the most significant problems in image processing, computer vision, and pattern recognition. Up to the present, two methodologies, model-free and model-based, have been proposed in segmentation approach [1]. In this paper, model means some parameters representing the shape of an object and constraints like continuity and smoothness.

Model-free algorithms [2][3] based on clustering, morphological filtering [4], and a watershed method [5] use local properties such as gray level, texture, and color. These local features can be useful. However these features are sensitive to noise and occlusion by other objects, not as expressive as global shape.

The model-based methods can generally find more accurate boundary of an object than model-free methods, but need more computational cost. The method proposed by Bhanu and Holben [6], extracts the shape by applying model information to the regions segmented by the local features. Kass et al. [7] introduced deformable contours to model complex shape. When the energy function is designed appropriately, their work performs well even if the boundary is deformed a little. Wang and Staib [8] used a point distribution model (PDM) [9] derived by the principal component analysis and employed the statistical shape distribution acquired from training sets. In case that enough training images describing the shape of an object are given, this method is relatively robust to noise and initial parameters than other model-based methods. In order to take full advantage of shape, our approach uses global shape information based on the point distribution model.

Considering only edge images extracted from several training images, the PDM algorithm models the global property of a shape. Also its Bayesian objective function, the measure to extract the boundary from an input image using the shape

\footnotetext{
* Correspondence: Email: chc@sdvision.kaist.ac.kr; WWW: http://sdvision.kaist.ac.kr; FAX: +82 0428698570
} 
model, depends on only the edge distribution of the input image. In some cases, the solution of the objective function for applying PDM to finding boundary fluctuates at the vicinity of the corner of an object, and this results in many iteration numbers for convergence.

The proposed method solves this problem by considering a curvature of the boundary. It models the global shape using curvature as well as edge of training images. This modeling method is based on the principal component analysis like the point distribution model. With the proposed shape model, we formulate the combined Bayesian objective function to find the unknown parameters representing the boundary. The objective function is derived using Bayesian rule. a prior is modeled by training images and the likelihood depends on edge and curvature of an input image and training images. Then we can extract the boundary by maximizing the objective function iteratively. The proposed method represents more detailed shape using curvature and finds the true position of high curvature points faster. So it can extract more accurate boundary and make the objective function converge rapidly.

We explain the modeling method of Point Distribution Model and the proposed modeling method for curvature model in the chapter 2, and derive the combined Bayesian objective function that are used in finding shape and pose parameters in the chapter 3. The experimental results are illustrated to comparison the performance of the proposed method with that of the conventional PDM in the chapter 4. In the last chapter, we describe the conclusion of this paper.

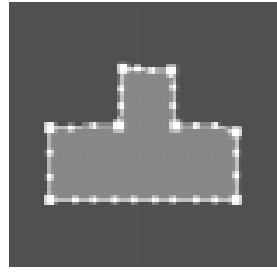

(a)

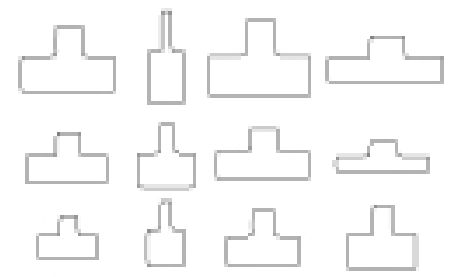

(b)

Fig. 1 Synthetic shape model by PDM (a) Synthetic image $(128 \times 128)$ with its 30 labeled points of the boundary, white dots are labeled points and bigger dots are critical labeled points. (b) 12 examples of synthetic shapes from a training set.

\section{STATISTICAL EDGE AND CURVATURE MODEL}

In order to employ global shape statistics obtained from training images, we have to model the shape with parameters. The edge and the curvature of the boundary are parameterized using the point distribution model and the proposed statistical curvature model, respectively.

\subsection{POINT DISTRIBUTION MODEL}

The point distribution model (PDM) [8] is useful for describing the shape. The PDM represents each shape of training images as shown in the figure 1(a). To determine the labeled points, we extract the critical points that have high curvature on the boundary. The critical points can represent the characteristic of a shape better than other points do. Then equally spaced points are interpolated between the critical points. Given $M$ aligned training shapes as the figure 1 (b), each of these is described by a position vector. The position vector is $\mathbf{L}_{i}=\left[x_{i}(1), y_{i}(1), x_{i}(2), y_{i}(2), \ldots, x_{i}(N), y_{i}(N)\right]^{\mathrm{T}}$ $(i=1, \ldots, M)$, where $N$ is the number of total labeled point in a training image. A mean shape, $\overline{\mathbf{L}}$, and a covariance with respect to the mean are calculated. According to the principal component analysis, any shape of the training set can be 
approximated using the mean shape and a weighted sum of deviations obtained from eigenvectors of the covariance matrix:

$$
\mathbf{L}=\overline{\mathbf{L}}+Q \mathbf{b},
$$

where $Q=\left(\mathbf{q}_{1}\left|\mathbf{q}_{2}\right| \ldots \mid \mathbf{q}_{t}\right)$ is the matrix of the first $t$ eigenvectors, and $\mathbf{b}=\left(b_{1}, b_{2}, \ldots, b_{t}\right)^{T}$ is weight vector. $\mathbf{b}$ indicates how much variation is exhibited with respect to each of the eigenvectors. This equation allows us to generate plausible shapes that are not part of the training set.

\subsection{STATISTICAL CURVATURE MODEL}

Using the same labeled points of the PDM as the figure 1(b), the statistical curvature model describes the curvature of the shape. The curvature statistics of the points are acquired from the aligned training images as the figure 1(a). The curvature of the boundary may be modeled with the principal component analysis like the equation (1). But this modeling method must evaluate additional numbers of unknown parameters like $\mathbf{b}$ because the eigenvectors obtained from the covariance matrix of the curvature are different from $Q$. Also this method causes the redundant processing to fit the two templates, the edge template and the curvature template, to the true boundary. This leads to heavy computational cost. In this paper, our aim is reduction of computational cost required to find the boundary. Observing edge and curvature with respect to the same deformed template that is the local contour described by $\mathbf{b}$, our method avoids the redundant computation and leads the faster convergence for optimizing the parameter, $\mathbf{b}$.

In the proposed approach, we define the curvature vector as

$$
\mathbf{R}_{i}=\left[r_{i}(1), r_{i}(2), \ldots, r_{i}(N)\right]^{T} \quad(i=1,2, \ldots, M),
$$

where $r_{i}(j)$ is curvature value of the $j$ th labeled point in the $i$ th training image. With respect to curvature, the mean and the standard deviation of the $j$ th labeled point are as follows

$$
\bar{r}(j)=\frac{1}{M} \sum_{i=1}^{M} r_{i}(j), \quad \sigma_{r}(j)=\sqrt{\frac{1}{M} \sum_{i=1}^{M}\left(r_{i}(j)-\bar{r}(j)\right)^{2}} .
$$

Then the mean curvature vector is

$$
\overline{\mathbf{R}}=[\bar{r}(1), \bar{r}(2), \ldots, \bar{r}(N)] .
$$

Given these statistics of curvature, we model a curvature image of a training set with noise-corrupted version:

$$
R=R_{\mathrm{L}=\overline{\mathbf{L}}+Q \mathbf{b}}+n
$$

A curvature image, $\mathrm{R}$, is modeled with one of curvature templates, $R_{\mathrm{L}}$, obtained from the boundary represented by the equation (1) and additive and independent noise, $n$. A plausible curvature image that is not part of the training set can be generated with curvature image obtained from a boundary image of the training set and noise. For using prior knowledge derived from training images, the noise is characterized with standard deviation and mean of the equation (3). Considering the curvature model and point distribution model together, we can calculate the similarity between a true boundary and the boundary obtained by our model.

\section{BAYESIAN OBJECTIVE FUNCTION}

In order to apply the shape model to the problem of boundary extraction, we derive the objective function. It measures the degree of fitting a deformable template, so called a deformable contour in other approaches, to true boundary of an object. For formulating the objective function used in the optimization of the shape and the pose parameters, Bayesian rule is applied. The prior knowledge is modeled by the assumption that the probability density of a noise follows 
Gaussian distribution. The likelihood is derived from a simple image noise model. Then we pose the problem in a maximum a posteriori Bayesian formulation.

In this chapter, we discuss two issues, the edge-based objective function [8] and the curvature-based objective function. The edge-based objective function is the conventional method to find boundary in point distribution model. It considers only the edge image obtained from an input image. So the solution about some critical points may fluctuate near to edge points of an object. It results in many iteration numbers for convergence of the function. To solve this defect, we propose the curvature-based objective function based on our curvature model. It is used in observing curvature of the boundary and constraining the curvature of the deformable template to follow the curvature statistics derived from training images. This constraint can help extracting rapidly the true critical point among edge points. So the proposed method makes the deformable template fit to the true boundary rapidly.

\subsection{EDGE-BASED OBJECTIVE FUNCTION}

In the point distribution model, the combined parameter is represented by $\mathbf{p}=\left(s, \theta, T_{x}, T_{y}, b_{1}, b_{2}, \ldots, b_{t}\right)$. The pose parameters are scale $(s)$, rotation $(\theta)$, and translation $\left(T_{x}, T_{y}\right)$. The point representation of the $n$th boundary points $(\mathrm{n}=0,1, \ldots, N-1)$ is

$$
\begin{aligned}
& x(\mathbf{p}, n)=s \cos \theta\left[\bar{x}_{n}+\sum_{k=1}^{t} Q_{2 n, k} b_{k}\right]-s \sin \theta\left[\bar{y}_{n}+\sum_{k=1}^{t} Q_{2 n+1, k} b_{k}\right]+T_{x} . \\
& y(\mathbf{p}, n)=s \sin \theta\left[\bar{x}_{n}+\sum_{k=1}^{t} Q_{2 n, k} b_{k}\right]+s \cos \theta\left[\bar{y}_{n}+\sum_{k=1}^{t} Q_{2 n+1, k} b_{k}\right]+T_{y}
\end{aligned}
$$

We assume that a prior follows a multivariate Gaussian density as in [1]. Then a prior probability density of the boundary described by $\mathbf{p}$ is expressed as

$$
\operatorname{Pr}(\mathbf{p})=\prod_{i=1}^{N} \operatorname{Pr}\left(p_{i}\right)=\prod_{i=1}^{N} \frac{1}{\sigma_{i} \sqrt{2 \pi}} e^{-\frac{\left(p_{i}-m_{i}\right)^{2}}{2 \sigma_{n}^{2}}},
$$

where $p_{i}$ is the $i$ th component of $\mathbf{p}, m_{i}$ is the mean of $p_{i}$, and $\sigma$ is the variance. About the pose parameters, the variance can be calculated from the training set alignment. The maximization problem of the a posteriori probability with respect to the parameters $\mathbf{p}$ is simplified to maximize [7]

$$
M_{\text {edge }}(\mathbf{p})=\sum_{j=1}^{t+4}\left[-\frac{\left(p_{i}-m_{i}\right)^{2}}{2 \sigma_{j}^{2}}\right]+\frac{1}{\sigma^{2}} \sum_{n=1}^{N} E(x(\mathbf{p}, n), y(\mathbf{p}, n)),
$$

where $E$ is edge image of an input image and $E(x, y)$ is edge magnitude of $(x, y)$ pixel. The first term is a prior probability and the second term is the likelihood probability. As shown in the equation (8), the likelihood probability depends on only edges of deformed template described by $\mathrm{x}(\mathbf{p}, n)$ and $\mathrm{y}(\mathbf{p}, n)(n=0,2, \ldots, \mathrm{N}-1)$.

\subsection{CURVATURE-BASED OBJECTIVE FUNCTION}

The goal of the curvature-based objective function is to find the most probable object considering an input curvature image. In terms of probabilities, we have to decide which curvature image, $R_{\mathrm{p}}$, described by a particular value of the parameter vector, $\mathbf{p}$, corresponds to the true curvature image $R$. It need to evaluate the probability of the curvature template with $\operatorname{Pr}\left(R_{\mathbf{p}} \mid R\right)$ and find the maximum over $\mathbf{p}$. This can be expressed using Bayes rule as 


$$
\operatorname{Pr}\left(R_{\max } \mid R\right)=\max _{\mathbf{p}} \operatorname{Pr}\left(R_{\mathbf{p}} \mid R\right)=\max _{\mathbf{p}} \frac{\operatorname{Pr}\left(R \mid R_{\mathbf{p}}\right) \operatorname{Pr}\left(R_{\mathbf{p}}\right)}{\operatorname{Pr}(R)},
$$

where $R_{\max }$ is the maximum a posteriori solution, $\operatorname{Pr}\left(R_{\mathrm{p}}\right)$ is a prior probability density of the curvature image $R_{\mathrm{p}}$ and $\operatorname{Pr}\left(R \mid R_{\mathbf{p}}\right)$ is the likelihood of the curvature image. Applying curvature operator such as curvature scale space (CSS) [11], $R_{\mathbf{p}}$ is directly obtained from the boundary template described by $\mathbf{p}$. Therefore, there is no unknown variable except $\mathbf{p}$ in $R_{p}$. $\operatorname{Pr}\left(R_{p}\right)$ can bias the boundary finder to search for a particular range of shape and pose. And it is equal to $\operatorname{Pr}(\mathbf{p})$ as the statistical curvature model in the chapter 2 . The curvature image $R$ is represented by noise corrupted version of $R_{\mathrm{p}}$. The noise is independent and additive: $R=R_{\mathbf{p}}+n$. Then $\operatorname{Pr}\left(R \mid R_{\mathbf{p}}\right)$ is equivalent to $\operatorname{Pr}\left(R=R_{\mathbf{p}}+n\right)$ or $\operatorname{Pr}\left(n=R-R_{\mathbf{p}}\right)$. The noise at each pixel $n(x, y)$ equals $R(x, y)-R_{\mathbf{p}}(x, y)$ and is governed by the probability density $\operatorname{Pr}(n)$. Assuming these events are independent about each point and $\operatorname{Pr}(n)$ follows Gaussian with zero mean and standard deviation $\sigma_{n}$, the likelihood is

$$
\begin{aligned}
\operatorname{Pr}\left(R \mid R_{\mathrm{p}}\right) & =\prod_{A} \operatorname{Pr}(n(x, y)) \\
& =\prod_{A} \frac{1}{\sqrt{2 \pi} \sigma_{n}} \exp \left(-\frac{\left(R(x, y)-R_{\mathrm{p}}(x, y)\right)^{2}}{2 \sigma_{n}^{2}}\right),
\end{aligned}
$$

where $A$ is a entire area of an image. Because $R_{\mathbf{p}}$ supports only along the boundary described by $\mathbf{p}$, it is not necessary to sum over the entire image area. By eliminating constant terms and taking the logarithm, we get the simple likelihood as

$$
\begin{aligned}
\operatorname{Pr}\left(R \mid R_{\mathbf{p}}\right) & =\sum_{A} \ln \frac{1}{\sqrt{2 \pi} \sigma_{n}}-\frac{1}{2 \sigma_{n}^{2}}\left(\sum_{A-A_{\mathbf{p}}}\left(R(x, y)-R_{\mathbf{p}}(x, y)\right)^{2},\right. \\
& \left.+\sum_{A_{\mathrm{p}}}\left(R(x, y)-R_{\mathbf{p}}(x, y)\right)^{2}\right)
\end{aligned}
$$

where $A_{\mathbf{p}}$ is the area of the contour defined by the boundary $(x(\mathbf{p}, n), \mathrm{y}(\mathbf{p}, n)),(n=1,2, \ldots, N)$, in the curvature image $R_{\mathbf{p}}$. $R(x, y)$ and $R_{\mathrm{p}}(x, y)$ are curvature values of a pixel $(x, y)$. Since $R_{\mathrm{p}}(x, y)$ is the curvature image obtained from the deformable template described by $\mathbf{p}$, we make a curvature value of $A_{\mathbf{p}}$ in a template $\left(R_{\mathbf{P}}\right)$ be the corresponding curvature mean of the equation (3) along the boundary and be zero everywhere else. The variation of the noise is substituted with the variation of the curvature model. By eliminating constant terms, we get the simple likelihood as

$$
\operatorname{Pr}\left(R \mid R_{\mathbf{p}}\right)=-\sum_{j=1}^{N} \frac{(R(x(\mathbf{p}, j), y(\mathbf{p}, j))-\bar{r}(j))^{2}}{2 \sigma_{r}^{2}(j)}
$$

We can expand a posteriori function for curvature images with the assumption of independent Gaussian noise at each pixel as

$$
M_{\text {curvature }}\left(R_{\mathbf{P}}\right)=\ln \operatorname{Pr}\left(R_{\mathbf{p}}\right)-\sum_{j=1}^{N} \frac{(R(x(\mathbf{p}, j), y(\mathbf{p}, j))-\bar{r}(j))^{2}}{2 \sigma_{r}^{2}(j)} .
$$

Now, considering both edge and curvature, we define new a posterior probability with edge image $E$ and curvature image $R$ of an input image. Assuming the events about edge and curvature are independent each other, a posterior probability follows 


$$
\operatorname{Pr}\left(t_{\mathbf{p}}, R_{\mathbf{p}} \text { input image }\right)=\operatorname{Pr}\left(t_{\mathbf{p}} \mid E\right) \cdot \operatorname{Pr}\left(R_{\mathbf{p}} \mid R\right)
$$

By the logarithm, the combined Bayesian objective function with respect to parameter $\mathbf{p}$ is derived as

$$
M_{c o m}(\mathbf{p})=2 \sum_{j=1}^{t+4}\left[-\frac{\left(p_{i}-m_{i}\right)^{2}}{2 \sigma_{j}^{2}}\right]+\sum_{n=1}^{N}\left[\frac{1}{\sigma_{n}^{2}} E(x(\mathbf{p}, n), y(\mathbf{p}, n))-\frac{\left(R(x(\mathbf{p}, n), y(\mathbf{p}, n))-\bar{c}_{j}\right)^{2}}{2 \sigma_{c}^{2}(j)}\right]
$$

The first term expresses a prior probability. It is a double of $\operatorname{Pr}(\mathbf{p})$ because $\operatorname{Pr}\left(\mathrm{R}_{\mathbf{p}}\right)$ is equal to the $\operatorname{Pr}(\mathbf{p})$. The combined object function implies that the true boundary is the deformable template that satisfies the following properties. Firstly, the template described by $\mathbf{p}$ is similar to the boundary reconstructed with PDM. Secondly, the pixels representing the template have high edge magnitude. Thirdly, the curvature of template follows the statistic value acquired from training images using the curvature model.

\section{EXPERIMENTAL RESULT}

In the experiments, the steepest decent methods [9] is used to optimize $M_{c o m}(\mathbf{p})$. And we used the average errors as evaluation criteria to show the performance of the proposed method. The boundary error in each labeled point of the deformable boundary template is calculated by finding the distance to a closest point on a true boundary.

The image $(128 \times 128)$ shown in the figure 2 (a) is a synthetic image where the target object is not a part of training set and is rotated and occluded partially by other objects. The initial deformable template represented by small white rectangles in the figure 2(a) was defined by the mean of the training set. To compare the performance of the proposed combined objective function with one of the edge-based objective function in the PDM [8], we evaluated both the average errors and the maximum errors in each iteration time. Our method and the PDM use the same deformable boundary template described by $\mathbf{p}$ and the number of high curvature points considered is generally less than that of edge points. Therefore the computational cost of the two methods is approximately equal in one iteration time. But the required iteration number for finding the accurate boundary was decreased by the proposed method as shown in the figure 2(d). The main cause of this result was that our method could find rapidly the true critical points having high curvature in an input image. However, the local template of PDM fluctuated in edges of the corner region. The final result of our method, the figure 2(b), shows that the proposed method is insensitive to rotation of an object and occlusion by other object. The snake [6] could not extract the precise boundary of the object as the figure 2(c). In this comparison, we can prove the effect of global shape information derived from training sets.

In the figure 3, the robustness to noise was measured by adding different amounts of zero mean Gaussian noise to the synthetic images. The figure 3(a) is an input image where the initial labeled points have been defined by the mean boundary of training set and Gaussian noise of variance 1000 has been added. Two images of the figure 3(b) are the edge image extracted by Canny edge detector and the final result of the proposed method. We added another noise with different variances to the figure 3(a). The figure 3(c) and 3(d) are the results when the variances of the Gaussian noise added are 2000 and 4000, respectively. The figure 3(e) shows that our method is insensitive to noise and particular occlusion by other object.

The figure 4 is experimental results of the proposed method when our method is applied our method to low-quality real image like Infrared images,. This figure shows that our work gives quite good final contours even if the input image has low-quality and the luminance of the object is similar to one of background. 
Finally, to compare the performance of the proposed method with the conventional methods, watershed algorithm [5] and Snake [7] were applied to the Infrared images. Watershed algorithm is one of model-free algorithms and Snake is one of model-based algorithms. The figure 5(a) is the result of watershed algorithm and the figure 5(b) is the result of Snake. These results show that the model-free methods are not adequate to find a boundary of an object in low-quality images. In case that a boundary of an object is indistinct or the strong edge of other objects exists near, the model-based method using only the constraints of contour such as continuity, bending energy, and smoothness, is difficult to get accurate boundary.

\section{CONCLUSION}

Given a training set that describes a desired object, we solve the problem of extracting the boundary of the object. The proposed method models curvature with statistical curvature model and formulates the curvature-based objective function that is derived using a maximum a posteriori criterion in Bayesian rule. The optimum solution of the combined objective function fits the deformed contour to the true boundary of an object. In the experimental results, we showed the robustness of our method to noise, occlusion, and rotation. Compared with conventional PDM, the computational cost is decreased. This work performs well in extracting a boundary of the object of which the structure is a rigid body or is composed of pieces of rigid bodies. When the initial parameters are too far away from the true boundary, the optimization may be trapped by local minima corresponding to nearby edges. So the research about the robustness to the effect of initialization is needed further.

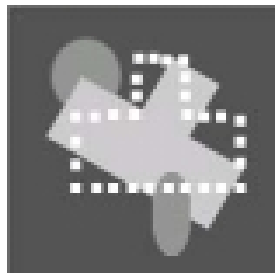

(a)

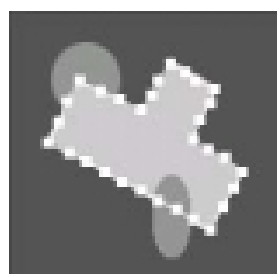

(b)

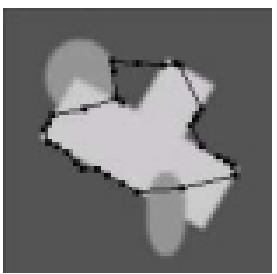

(c)

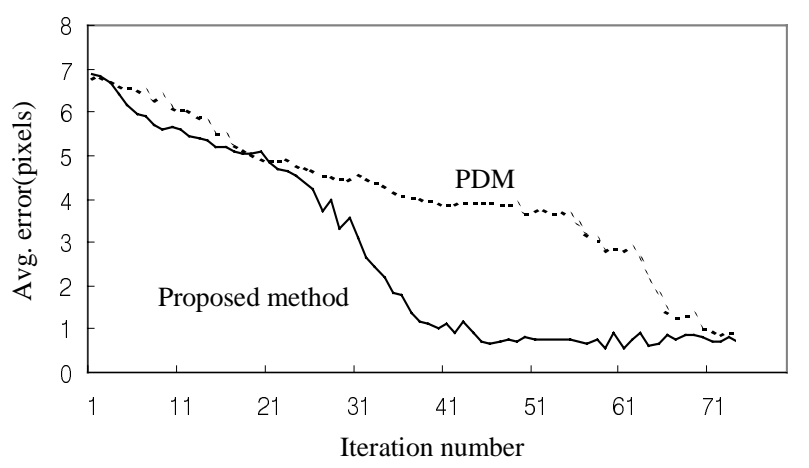

(d)

Fig. 2. Iteration number for convergence. (a) Initial labeled points and the input image. (b) Final result by the proposed method. (c) Final result of Snake [7]. (d) Mean error measure with respect to iteration numbers of the conventional PDM and the proposed algorithm. 


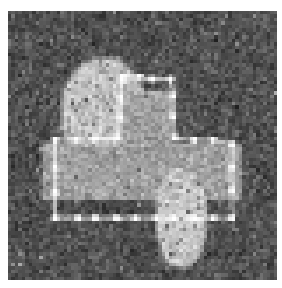

(a)
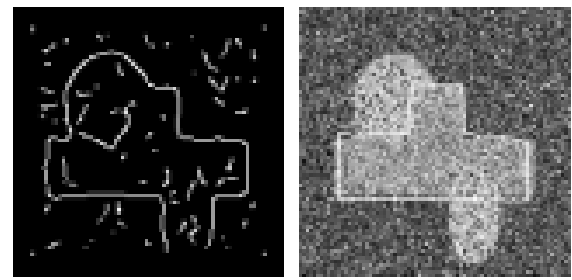

(c)
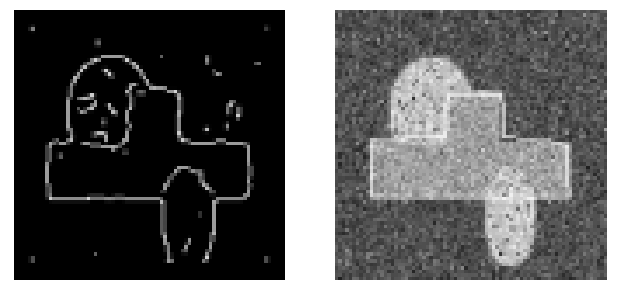

(b)
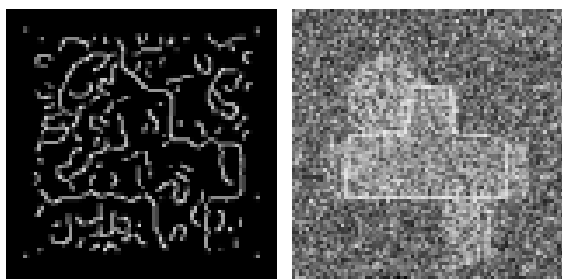

(d)

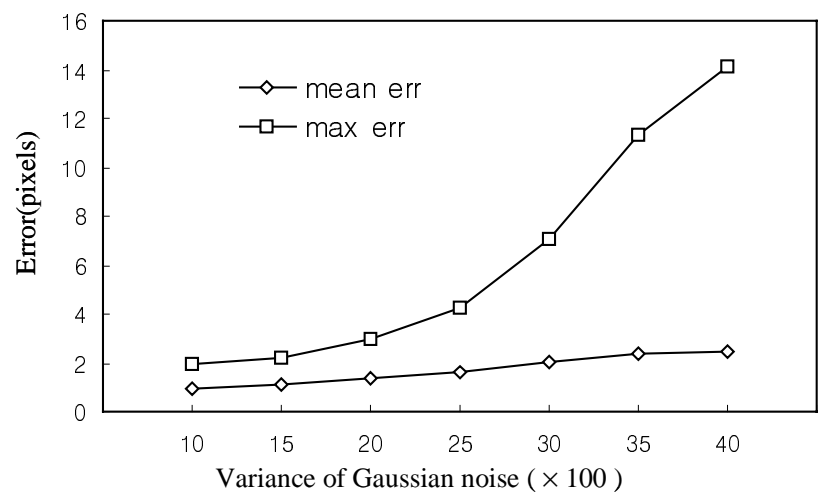

(e)

Fig. 3. Sensitivity to noise experiment. (a) Input image and initial labeled points. (b), (c), (d) Edge image and final boundary from (a) with Gaussian noise of that variance is 1000, 2000, 4000. (e) Average error and maximum error
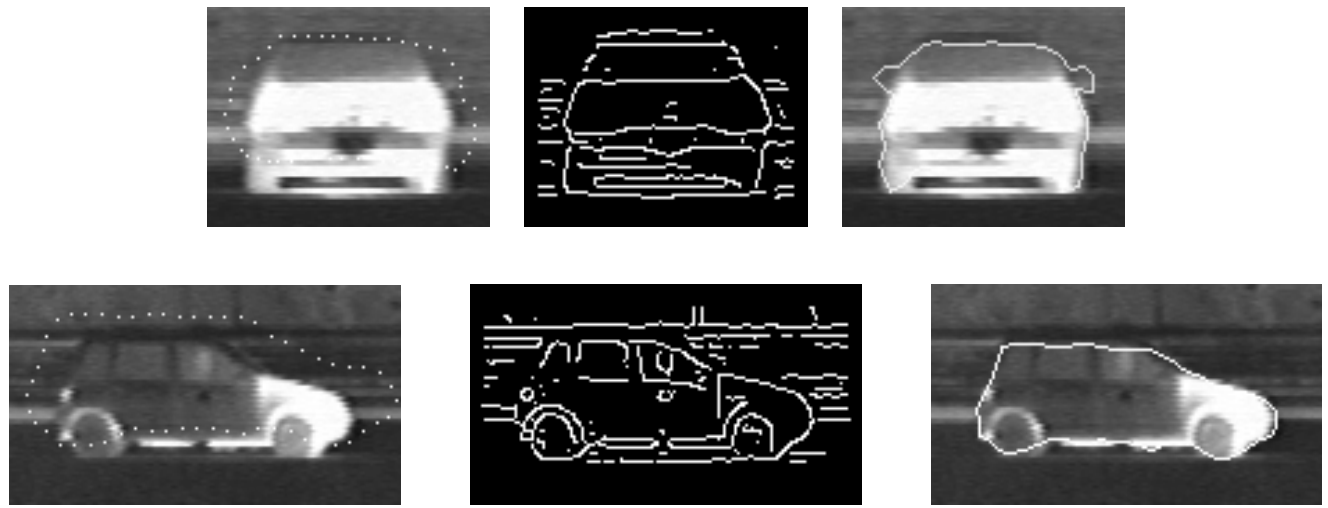

Fig. 4. The results of the proposed method applied to Infrared images. (left) Input image and initial contour. (middle) Canny edge image. (right) Final contour. 


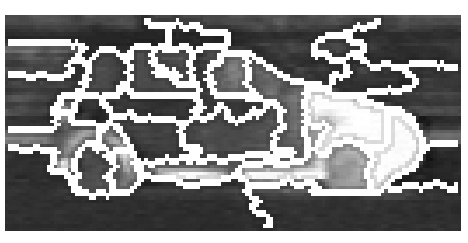

(a)

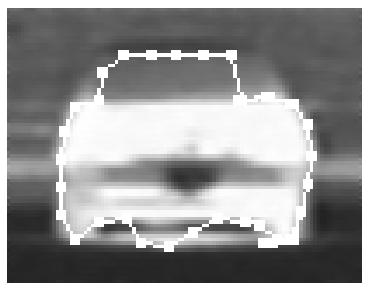

(b)

Fig. 5. The experimental results using: (a) watershed algorithm, (b) snake algorithm.

\section{REFERENCES}

1. L. H. Staib, and J. S. Duncan, "Boundary Finding with Parametrically Deformable Models," IEEE Trans. Pattern Analysis and Machine Intelligence, Vol. 14, No. 11, pp. 1061 1075, 1992.

2. P. K. Sahoo, S. Soltani, and A. K. C. Wong, “A Survey of Thresholding Techniques,” CVGIP, Vol. 41, pp.233 260, 1988.

3. Robert M. HarallickK and Linda G. Shapiro, "Survey of Image Segmentation Techniques," Computer Vision, Graphics, and Image Processing, Vol. 29, pp.100 132, 1985.

4. P. Salembier and M. Pardas, "Hierarchical Morphological Segmentation for Image Sequence Coding," IEEE. Trans. Image Processing, Vol. 3, No. 5, pp. 639 651, Sep. 1994.

5. L. Vincent and P. Soille, "Watersheds in Digital Spaces: An Efficient Algorithm Based on Immersion Simulation," IEEE Trans. Pattern Analysis and Machine Intelligence, Vol. 13, No. 6, pp. 583 598, June 1991.

6. B. Bhanu, R.D. Holben, "Model-based Segmentation of FLIR Images," IEEE Trans. Aerospace and Electronic System, Vol. 26, No. 1, pp. 2 10, 1990.

7. M. Kass, A. Witkin, D. Terzopoulos, "Snakes : Active Contour Model", International Journal of Computer Vision, pp. $321 \sim 331,1988$.

8. Y. Wang, and L.H. Staib, "Boundary Finding with Prior Shape and Smoothness Methods," IEEE Trans. Pattern Analysis and Machine Intelligence, Vol. 22, No. 7, pp. 738 743, July 2000.

9. M. Sonka, V. Hlavac, R. Boyle, Image Processing, Analysis, and Machine Vision, PWS publishing, 1999.

10. J. F. Canny, "a Computational Approach to Edge-detection," IEEE Trans. Pattern Anyaysis and Machine Intelligence, Vol. 8, No. 6, pp. 679-698, 1986.

11. F. Mokhtarian, R. Suomela, "Curvature Scale Space for Image Point Feature Detection," in Proceedings of 7th International Conference on Image Processing, Vol. 1, pp. 206-210, 1999. 